\title{
Nonperturbative Treatment of non-Markovian Dynamics of Open Quantum Systems
}

\author{
D. Tamascelli, ${ }^{1,2}$ A. Smirne, ${ }^{2}$ S. F. Huelga, ${ }^{2}$ and M. B. Plenio ${ }^{2}$ \\ ${ }^{1}$ Università degli Studi di Milano, Dipartimento di Fisica, Via Celoria 16, I-20133 Milano, Italy \\ ${ }^{2}$ Institute of Theoretical Physics, Universität Ulm, Albert-Einstein-Allee 11D-89069 Ulm, Germany
}

(Received 15 September 2017; published 19 January 2018)

\begin{abstract}
We identify the conditions that guarantee equivalence of the reduced dynamics of an open quantum system (OQS) for two different types of environments - one a continuous bosonic environment leading to a unitary system-environment evolution and the other a discrete-mode bosonic environment resulting in a system-mode (nonunitary) Lindbladian evolution. Assuming initial Gaussian states for the environments, we prove that the two OQS dynamics are equivalent if both the expectation values and two-time correlation functions of the environmental interaction operators are the same at all times for the two configurations. Since the numerical and analytical description of a discrete-mode environment undergoing a Lindbladian evolution is significantly more efficient than that of a continuous bosonic environment in a unitary evolution, our result represents a powerful, nonperturbative tool to describe complex and possibly highly non-Markovian dynamics. As a special application, we recover and generalize the well-known pseudomodes approach to open-system dynamics.
\end{abstract}

DOI: $10.1103 /$ PhysRevLett.120.030402

Introduction.-Each and every realization of physical quantum systems will unavoidably suffer from interactions with uncontrollable degrees of freedom, namely, the surrounding environment. In certain situations, it is possible to model such an interaction as resulting in white noise, amenable to an effective description in terms of a Lindblad master equation [1-4]. In general, however, the relevant noise sources originating from the interaction with structured environments give rise to non-Markovian effects and call for a more complex characterization [5-7]. Solidstate implementations of qubits [8-10], nanoscale quantum thermal machines [11,12], sensing and metrology $[13,14]$, energy-charge conversion and exciton transport in solidstate devices $[15,16]$, or biological light harvesting complexes $[17,18]$ are typical instances in which deviations from a Lindbladian evolution can play a significant role.

The simulation of even simple OQSs interacting with structured environments is a formidable task. If one adopts state-of-the-art numerical methods [19-22] for the simulation of the dynamics of a quantum system, only systems of a few qubits are accessible. A similar situation arises in proposed quantum physical simulators of systemenvironment interaction [23]. The difficulty, in both cases, is due to the large number of environmental degrees of freedom affecting the reduced dynamics of the OQS under investigation. Several approaches have been developed to map the original model into a unitarily equivalent one, which is easier to deal with, e.g., because it possesses a more suitable configuration for the application of proper numerical techniques [21,24-27]. Yet, the simulation of such equivalent unitary models remains challenging since the number of environmental degrees of freedom involved is essentially unchanged.
On the other hand, if one is actually interested in the evolution of the open system only, it is clear that the problem would be simplified drastically by finding simpler auxiliary systems, which might not be directly related to the original ones at the level of the overall dynamics, but which yield the same reduced dynamics for the open system. In this regard, a powerful idea is to decompose the action of the environment into a non-Markovian core which, in turn, interacts with a Markovian environment. The former interacts coherently with the open system and encloses all the memory effects during the evolution, while the latter can be characterized effectively by a Lindblad equation and represents the residual unidirectional leaking of information out of the non-Markovian core [28-34] (see Fig. 1). Of course, the most appealing feature of this approach is that the resulting configuration will be generally much simpler than the original unitary one, having to deal with a considerably smaller number of degrees of freedom.

The possibility to reproduce the reduced OQS dynamics obtained from a unitary evolution involving a complex environment via a simpler environment, which itself is subject to a Lindblad dynamics, is usually supported by a good agreement with experimental data or numerical analysis, as well as by approximative arguments, which can be applied in certain specific regimes. Nevertheless, rigorous results or theorems of some generality are still lacking, and the only exact result was derived in [35] for the spin-boson model, with a specific form of the interaction and a zero-temperature environment. There, a procedure was introduced to replace the environment with infinitely many degrees of freedom by a finite set of auxiliary harmonic modes, the so-called pseudomodes, which 


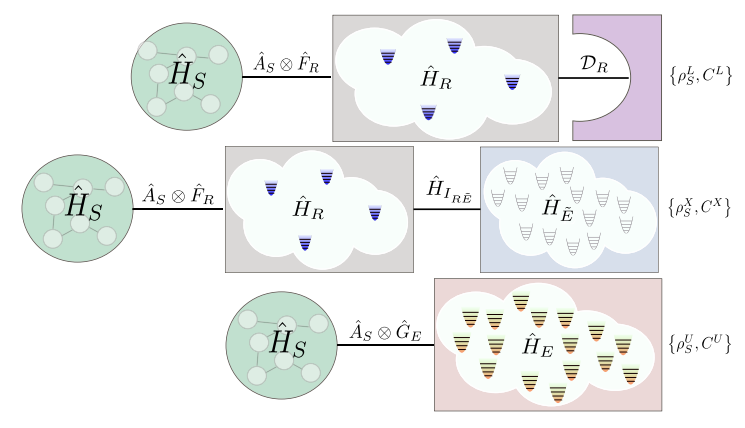

FIG. 1. Graphical representation of the considered configurations. Upper diagram: A possibly composite system interacting with the environment $R$ undergoing the Lindblad dynamics as defined in Eq. (2). Lower: Same system interacting with the unitarily evolving environment $E$ [see Eq. (7)]. Middle: The environment $R$ has been extended as to include additional modes with free evolution $H_{\tilde{E}}$ and interacting with the $R$ modes through $\hat{V}_{R \tilde{E}}$ [see Eq. (11)].

proved to be a very useful tool to characterize openquantum-system dynamics [36-45].

In this Letter, we provide a proof of the general equivalence between the reduced dynamics of an OQS interacting unitarily with a bosonic environment and the dynamics of the same OQS interacting with a typically much simpler harmonic environment subject to a Lindblad evolution. We prove that, for initial Gausssian states of the environments, the equivalence is guaranteed if the environmental expectation values and two-time correlation functions of the two configurations are equal for all times. We stress that, while this is well known if one compares the reduced dynamics of two unitary evolutions [2,46-48], it is a priori not obvious that the same still applies when comparing two reduced dynamics obtained from a unitary and a nonunitary Lindbladian evolution. The result holds irrespective of the strength of the system-environment interaction or the structure of the environment, thus providing a general nonperturbative way to describe OQS dynamics, possibly highly non-Markovian ones. As a special case, we recover the equivalence between the reduced dynamics of the spin-boson model and the description given by the pseuodomodes, directly generalizing it to different forms of the coupling.

The main result.-We start by introducing the nonunitary configuration, which consists of a quantum system $S$ interacting with a bosonic environment $R$, which is, in turn, subject to a Lindblad evolution. The system-environment Hamiltonian reads

$$
\hat{H}_{S R}=\hat{H}_{S}+\hat{H}_{R}+\sum_{j=1}^{\kappa} \hat{A}_{S, j} \otimes \hat{F}_{R, j},
$$

where, for a system of dimension $d_{S}, \kappa$ can take values in $1,2, \ldots, d_{S}^{2}$. Here and in what follows, we imply the tensor product with the identity so that $\hat{H}_{S}$ will be used instead of $\hat{H}_{S} \otimes \mathbb{1}$ and so on. The Lindbladian dynamics of the bipartite system $S-R$ is fixed by the master equation $(\hbar=1)$

$\dot{\rho}_{S R}(t)=\mathcal{L}_{S R}\left[\rho_{S R}(t)\right]=-i\left[\hat{H}_{S R}, \rho_{S R}(t)\right]+\mathcal{D}_{R}\left[\rho_{S R}(t)\right]$,

where

$$
\mathcal{D}_{R}[\rho]=\sum_{j=1}^{\ell} \gamma_{j}\left(\hat{L}_{R, j} \rho \hat{L}_{R, j}^{\dagger}-\frac{1}{2}\left\{\hat{L}_{R, j}^{\dagger} \hat{L}_{R, j}, \rho\right\}\right)
$$

acts on $R$ only, and $\ell$ determines the number of degrees of freedom of $R$ and hence, the complexity of the nonunitary model. We consider time-independent arbitrary Lindblad operators $\hat{L}_{R, j}$ and coefficients $\gamma_{j} \geq 0$, ensuring the complete positivity of the evolution $[2,49]$ and a factorized initial state $\rho_{S R}(0)=\rho_{S}(0) \otimes \rho_{R}(0)$. We denote by $\rho_{S}^{L}(t)$ the corresponding reduced state of the system $S$ at time $t$, namely,

$$
\rho_{S}^{L}(t)=\operatorname{Tr}_{R}\left\{e^{\mathcal{L}_{S R} t}\left[\rho_{S}(0) \otimes \rho_{R}(0)\right]\right\} .
$$

Here, $F_{R, j}(t)$ are the expectation values of the $R$ interaction operators with respect to the "free" evolution of the environment $R$ (i.e., without taking into account the presence of the system $S), F_{R, j}(t)=\operatorname{Tr}_{R}\left\{\hat{F}_{R, j} e^{\mathcal{L}_{R} t}\left[\rho_{R}(0)\right]\right\}$, where $\mathcal{L}_{R}$ is the generator

$$
\mathcal{L}_{R}[\rho]=-i\left[\hat{H}_{R}, \rho\right]+\mathcal{D}_{R}[\rho]
$$

Moreover, we denote by

$$
\left.C_{j j^{\prime}}^{L}(t+s, s)=\operatorname{Tr}_{R}\left\{\hat{F}_{R, j} e^{\mathcal{L}_{R} t\left[\hat{F}_{R, j^{\prime}}\right.} e^{\mathcal{L}_{R} s}\left[\rho_{R}(0)\right]\right]\right\}
$$

the environment two-time correlation functions.

The other configuration we consider is given by the same system $S$ interacting unitarily with a bosonic environment $E$. The unitary $S-E$ evolution is determined by the Hamiltonian

$$
\hat{H}_{S E}=\hat{H}_{S}+\hat{H}_{E}+\sum_{j=1}^{\kappa} \hat{A}_{S, j} \otimes \hat{G}_{E, j}
$$

and the initial factorized state $\rho_{S E}(0)=\rho_{S}(0) \otimes \rho_{E}(0)$. We denote with $\rho_{S}^{U}(t)$ the corresponding $S$ reduced state at time $t$; i.e.,

$$
\rho_{S}^{U}(t)=\operatorname{Tr}_{E}\left\{e^{-i \hat{H}_{S E} t}\left[\rho_{S}(0) \otimes \rho_{E}(0)\right] e^{i \hat{H}_{S E} t}\right\},
$$

the expectation values of the interaction terms as $G_{E, j}(t)=$ $\operatorname{Tr}_{E}\left\{\hat{G}_{E, j} e^{-i \hat{H}_{E} t} \rho_{E}(0) e^{i \hat{H}_{E} t}\right\}$, and with $C_{j j^{\prime}}^{U}(t+s, s)$, the two-time correlation functions

$$
\begin{aligned}
C_{j j^{\prime}}^{U}(t+s, s)= & \operatorname{Tr}_{E}\left\{e^{i \hat{H}_{E}(t+s)} \hat{G}_{E, j} e^{-i \hat{H}_{E}(t+s)}\right. \\
& \left.e^{i \hat{H}_{E} s} \hat{G}_{E, j^{\prime}} e^{-i \hat{H}_{E} s} \rho_{E}(0)\right\}
\end{aligned}
$$

Theorem.-Given the two systems described by Eqs. (1)-(6) and Eqs. (7)-(9), respectively, if both $\rho_{R}(0)$ and $\rho_{E}(0)$ are Gaussian states, then the following implication holds: 


$$
\begin{aligned}
& \left.\begin{array}{rl}
F_{R, j}(t) & =G_{E, j}(t) \\
C_{j j^{\prime}}^{L}(t+s, s) & =C_{j j^{\prime}}^{U}(t+s, s)
\end{array}\right\} \quad \forall j, j^{\prime}, t, s \geq 0 \\
& \Rightarrow \rho_{S}^{L}(t)=\rho_{S}^{U}(t) \quad \forall t .
\end{aligned}
$$

Sketch of the Proof.-The proof relies on two main steps, which we represent as separate lemmas. Lemma 1 establishes a dilation [3,50-52] of the Lindblad equation on the bipartite system $S-R$ in Eq. (2) to a unitary evolution on a tripartite system $S-R-\tilde{E}$ such that the exact reduced dynamics of $S$ under the latter is equal to that under the Lindbladian dynamics on $S-R$. Because of that, we can translate the comparison between the two open-system dynamics of the configurations we are interested in into a comparison between the open-system dynamics of $S$ obtained from the unitaries on $S-E$ and $S-R-\tilde{E}$, respectively. These two open-system dynamics can be easily shown to be equivalent if their environmental expectation values and two-time correlation functions are equal (as we show in the last part of the proof). But now Lemma 2 shows that the two-time correlation functions of operators on $R$ with respect to the unitary dynamics on $R-\tilde{E}$ are equal to those obtained via the reduced Lindbladian dynamical maps on $R$ [53], i.e., to $C_{j j^{\prime}}^{L}(t+s, s)$, while the analogous correspondence between the expectation values is implied directly by Lemma 1 . Hence, we get the implication stated in the Theorem. Figure 1 represents the different configurations involved in the proofs of the Lemmas and the Theorem, which are given in the Supplemental Material [55].

Lemma 1. Consider a tripartite system $S-R-\tilde{E}$ undergoing the unitary evolution fixed by

$$
\begin{aligned}
\hat{H}_{S R \tilde{E}} & =\hat{H}_{S R}+\hat{H}_{\tilde{E}}+\hat{V}_{R \tilde{E}}, \\
\hat{H}_{\tilde{E}} & =\sum_{j=1}^{\ell} \int_{-\infty}^{\infty} d \omega \omega \hat{b}_{\tilde{E}}^{\dagger}(\omega, j) \hat{b}_{\tilde{E}}(\omega, j), \\
\hat{V}_{R \tilde{E}} & =\sum_{j=1}^{\ell} \sqrt{-\frac{\gamma_{j}}{2 \pi}} \int_{-\infty}^{\infty} d \omega \hat{L}_{R, j} \hat{b}_{\tilde{E}}^{\dagger}(\omega, j)-\hat{L}_{R, j}^{\dagger} \hat{b}_{\tilde{E}}(\omega, j),
\end{aligned}
$$

where $\hat{b}_{\tilde{E}}(\omega, j)$ and $\hat{b}_{\tilde{E}}^{\dagger}(\omega, j)$ are bosonic annihilation and creation operators of a Fock space $\mathcal{H}_{\tilde{E}, j}$, $\left[\hat{b}_{\tilde{E}}(\omega, j), \hat{b}_{\tilde{E}}^{\dagger}\left(\omega^{\prime}, j^{\prime}\right)\right]=\delta_{j j^{\prime}} \delta\left(\omega-\omega^{\prime}\right)$, and the global Fock space associated with the environment $\tilde{E}$ is indeed the tensor product $\mathcal{H}_{\tilde{E}}=\otimes_{j=1}^{\ell} \mathcal{H}_{\tilde{E}, j}$. Let the initial state be $\rho_{S R \tilde{E}}(0)=\rho_{S R}(0) \otimes\left|0_{\tilde{E}}\right\rangle\left\langle 0_{\tilde{E}}\right|$, where $\left|0_{\tilde{E}}\right\rangle=\otimes_{j=1}^{\ell}\left|0_{j}\right\rangle$ is the vacuum state of $\mathcal{H}_{\tilde{E}}$. Moreover, denote as $\rho_{S R}^{X}(t)$ the reduced $S-R$ state at time $t$; i.e.,

$\rho_{S R}^{X}(t)=\operatorname{Tr}_{\tilde{E}}\left\{e^{-i \hat{H}_{S R \tilde{E}} t}\left(\rho_{S R}(0) \otimes\left|0_{\tilde{E}}\right\rangle\left\langle 0_{\tilde{E}}\right|\right) e^{i \hat{H}_{S R \tilde{E}} t}\right\} ;$

then [still denoting with $\rho_{S R}(t)$ the state fulfilling Eq. (2)],

$$
\rho_{S R}^{X}(t)=\rho_{S R}(t) \text {. }
$$

Lemma 2. Given the unitary dynamics on $R-\tilde{E}$ fixed by the Hamiltonian $\hat{H}_{R \tilde{E}}=\hat{H}_{R}+\hat{H}_{\tilde{E}}+\hat{V}_{R \tilde{E}}$, see Eq. (11), its correlation functions

$$
\begin{aligned}
C_{j j^{\prime}}^{X}(t+s, s)= & \operatorname{Tr}_{R E}\left\{e^{i \hat{H}_{R \tilde{E}}(t+s)} \hat{F}_{R, j} e^{-i \hat{H}_{R \tilde{E}}(t+s)}\right. \\
& \left.e^{i \hat{H}_{R \tilde{E}} s} \hat{F}_{R, j^{\prime}} e^{-i \hat{H}_{R \tilde{E}^{s}}}\left[\rho_{R}(0) \otimes \rho_{\tilde{E}}(0)\right]\right\}
\end{aligned}
$$

satisfy

$$
C_{j j^{\prime}}^{X}(t+s, s)=C_{j j^{\prime}}^{L}(t+s, s) \quad \forall t, s \geq 0 .
$$

The Theorem shows that if we fix the open system $S$, i.e., its free dynamics as given by $\hat{H}_{S}$ and how it interacts with the environment as given by the $\hat{A}_{S, j}$, the equivalence of the expectation values $F_{R, j}(t)$ and $G_{E, j}(t)$ and of the correlation functions $C_{j j^{\prime}}^{L}(t+s, s)$ and $C_{j j^{\prime}}^{U}(t+s, s)$ ensures the equivalence of the open-system dynamics of the two configurations. On the other hand, the environmental Hamiltonians $\hat{H}_{R}$ and $\hat{H}_{E}$ need not be equal, neither do the environmental interaction operators $\hat{F}_{R, j}$ and $\hat{G}_{E, j}$ or the initial Gaussian states $\rho_{R}(0)$ and $\rho_{E}(0)$, nor are we setting a priori any constraint on the form of the Lindblad operators $\hat{L}_{R, j}$.

Crucially, the equivalence between the two reduced dynamics, governed by Eqs. (1)-(6) and Eqs. (7)-(9), respectively, can be then guaranteed in the presence of a Lindbladian environment $R$, which is much simpler than the unitary one $E$, being characterized by a considerably smaller number of degrees of freedom. We will give an explicit example in the next paragraph. We also emphasize that the Theorem provides us with a manifestly nonperturbative way to deal with general, non-Markovian open-system dynamics since we have not set any restriction on the strength of the coupling, nor on the structure of the environment. In addition, our result can be straightforwardly generalized beyond the assumption of initial Gaussian states by asking for the equality of the higherorder correlation functions of the environments $R$ and $E$, see [55]. Finally, we stress that our results in their present form establish the equivalence between the reduced dynamics of the unitary and the Lindbladian scenarios for singletime expectation values of open-system observables while the case of multitime correlators remains open.

The spin-boson model and the pseuodomodes.Consider a two-level system coupled to an environment of harmonic oscillators with a Hamiltonian as in Eq. (7), where the interaction is in bilinear form [2,56]. We use $\sigma_{i}$, $i=x, y, z$ to denote the Pauli matrices, $\sigma_{+}=\sigma_{-}^{\dagger}=|1\rangle\langle 0|$, with $|1\rangle,|0\rangle$ eigenvectors of $\sigma_{z}$, while $\hat{a}_{\omega}$ and $\hat{a}_{\omega}^{\dagger}$ are the annihilation and creation operators of the bosonic field, $\left[\hat{a}_{\omega}, \hat{a}_{\omega^{\prime}}^{\dagger}\right]=\delta\left(\omega-\omega^{\prime}\right)$. In particular, we take $\hat{H}_{S}=\omega \sigma_{z}$ and $\hat{H}_{E}=\int_{-\infty}^{+\infty} d \omega \omega \hat{a}_{\omega}^{\dagger} \hat{a}_{\omega}$, while we consider two different forms of interaction. In one case, we set $\hat{A}_{S}=\sigma_{x}$ and $\hat{G}_{E}=\int_{-\infty}^{\infty} d \omega\left(g(\omega) \hat{a}_{\omega}+g^{*}(\omega) \hat{a}_{\omega}^{\dagger}\right)$, while in the other, we take two terms in the interaction (we use primed 
letters to denote the corresponding interaction operators) $\hat{A}_{S, 1}^{\prime}=\sigma_{-}, \quad \hat{A}_{S, 2}^{\prime}=\sigma_{+}, \quad \hat{G}_{E, 1}^{\prime}=\int_{-\infty}^{\infty} d \omega g(\omega) \hat{a}_{\omega}, \quad$ and $\hat{G}_{E, 2}^{\prime}=\hat{G}_{E, 1}^{\prime \dagger}$. Note that the second form of the coupling can be obtained from the former after the rotating wave approximation, and, importantly, it conserves the total number of excitations [2,57]. Restricting for simplicity to a zero-temperature environment, $\rho_{E}(0)=|0\rangle\langle 0|$, which is stationary with respect to $\hat{H}_{E}$, the expectation values of the environmental interaction operators vanish, and there is only one nontrivial two-time correlation function for both versions of the system-environment coupling, namely,

$$
\begin{aligned}
C_{S B}^{U}(t) & =\operatorname{Tr}_{E}\left\{e^{i \hat{H}_{E} t} \hat{G}^{\prime}{ }_{E, 1} e^{-i \hat{H}_{E} t} \hat{G}^{\prime}{ }_{E, 2}|0\rangle\langle 0|\right\} \\
& =\int_{-\infty}^{\infty} d \omega \mathcal{S}(\omega) e^{-i \omega t},
\end{aligned}
$$

where we introduced $\mathcal{S}(\omega)=|g(\omega)|^{2}$, usually referred to as correlation spectrum [58].

Let us now consider the nonunitary dynamics fixed by Eqs. (1)-(3), with the same $\hat{H}_{S}$ and $\hat{A}_{S, j}$ specified above, and where $R$ is defined via a set of independent auxiliary harmonic modes, with the annihilation and creation operators $\hat{c}_{j}$ and $\hat{c}_{j}^{\dagger}, j=1, \ldots \ell$ such that $\left[\hat{c}_{j}, \hat{c}_{l}^{\dagger}\right]=\delta_{j l}$. Furthermore, we set $\hat{H}_{R}=\sum_{j=1}^{\ell} \eta_{j} \hat{c}_{j}^{\dagger} \hat{c}_{j}$, and we still consider two forms of the coupling, $\hat{F}_{R}=\sum_{j=1}^{\ell}\left(\lambda \hat{c}_{j}+\right.$ $\lambda^{*} \hat{c}_{j}^{\dagger}$ ) (together with $\hat{A}_{S}=\sigma_{x}$ ) or $\hat{F}_{R, 1}^{\prime}=\sum_{j=1}^{\ell} \lambda \hat{c}_{j}$ and $\hat{F}_{R, 2}^{\prime}=\hat{F}_{R, 1}^{\prime \dagger}$ (together with $\hat{A}_{S, 1}^{\prime}=\hat{A}_{S, 2}^{\prime \dagger}=\sigma_{+}$). The Lindblad generator acting on $R$ is defined as in Eq. (5), with dissipator

$$
\mathcal{D}_{R}[\rho]=\sum_{j=1}^{\ell} \gamma_{j}\left(\hat{c}_{j} \rho \hat{c}_{j}^{\dagger}-\frac{1}{2}\left\{\hat{c}_{j}^{\dagger} \hat{c}_{j}, \rho\right\}\right) .
$$

Fixing $\rho_{R}(0)=|0\rangle\langle 0|$, which is stationary with respect to $\mathcal{L}_{R}$, for both the forms of the coupling, the environmental expectation values vanish, and there is still only one nontrivial two-time correlation function, see Eq. (6), given by

$$
\begin{aligned}
C_{S B}^{L}(t) & =\operatorname{Tr}_{R}\left\{\hat{F}^{\prime}{ }_{R, 1} e^{\left.\mathcal{L}_{R} t\left[\hat{F}^{\prime}{ }_{R, 2}|0\rangle\langle 0|\right]\right\}}\right. \\
& =|\lambda|^{2} \sum_{j=1}^{\ell} e^{\left(i \eta_{j}-\gamma_{j} / 2\right) t} .
\end{aligned}
$$

We conclude that for any unitary spin-boson dynamics such that the Fourier transform of the spectrum $S(\omega)$, see Eq. (16), can be written as a sum of $\ell$ exponentials as in Eq. (18), we can define an equivalent nonunitary dynamics, which only involves $\ell$ modes and which yields the same reduced dynamics on the two-level system $S$ at any time. For the excitation-preserving form of the coupling, this is equivalent to the result obtained in [35], and the $\ell$ modes of the environment $R$ precisely identify with the pseudomodes introduced there. Hence, not only have we recovered this result as a direct application of our Theorem, but we have also generalized it to a different form of the system-environment coupling, not preserving the number of excitations.

To give an explicit example, let us consider the Lindblad equation for a single harmonic oscillator, interacting via the excitation nonpreserving coupling with the two-level system and damped as in Eq. (17), with $\ell=1$. For any choice of the defining parameters $\lambda, \eta_{1}$, and $\gamma_{1}$, the reduced dynamics of the qubit interacting with the damped harmonic oscillator will be the same as the reduced dynamics of a qubit interacting with infinitely many harmonic oscillators, with correlation spectrum given by the Lorentzian $\mathcal{S}(\omega)=|\lambda|^{2} \gamma_{1} /\left[\left(\gamma_{1}^{2}+\left(\omega-\eta_{1}\right)^{2}\right)\right]$, see Eq. (18). In Fig. 2, we show an example of these two reduced dynamics of the two-level system. In particular, the parameters have been chosen as to have the mode at resonance with the qubit $\left(\eta_{1}=\omega\right)$ and the strong coupling regime $\left(\lambda>\gamma_{1}\right)$. Note that such a regime can be realized in an ion-trap setup [34]. Moreover, the part of the correlation spectrum on the negative frequencies (which is fixed by the ratio $\left.\gamma_{1} / \eta_{1}\right)$ is not negligible, which provides us with a regime where the approximative argument put forward in [28] does not apply. The unitary evolution has been simulated using the numerically exact TEDOPA algorithm [21]. The two curves in the graphs overlap perfectly. This exemplifies the equivalence between an environment with a Lorentzian spectrum and an environment consisting of a damped harmonic oscillator in a setting where neither the original pseudomode approach [35] nor the approximated argument of [28] would apply.

The simple example now described allows us to emphasize another important issue. If we want to restrict the definition of the environment to the positive frequencies only, we set (for both forms of the coupling) $g(\omega)=0$ for $\omega<0$, and hence, $\mathcal{S}(\omega)=0$ for $\omega<0$. The latter condition can be also easily seen to follow from the fluctuationdissipation relation [2] applied to a zero-temperature environment. Now, no finite number of auxiliary modes can reproduce exactly a two-time correlation function, whose

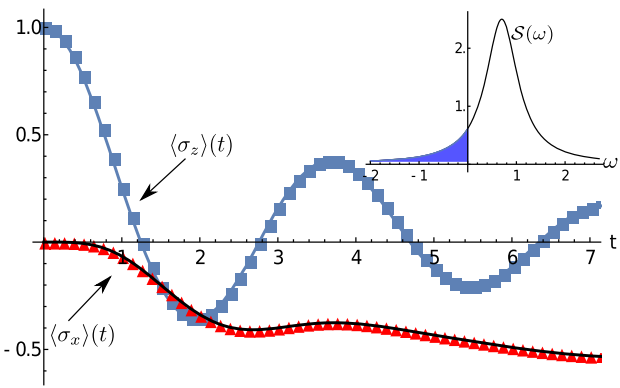

FIG. 2. Comparison between the expectation values of the observables $\sigma_{z}$ and $\sigma_{x}$ on the reduced state $\rho_{S}(t)$ as obtained by Lindblad (solid lines) and unitary (TEDOPA) dynamics (blue squares and red triangles). The parameters are $\omega=\eta_{1}=0.7$, $\lambda=0.6, \gamma_{1}=0.4$. The Lorentzian correlation spectrum $\mathcal{S}(\omega)$ for this parametrization is shown in the inset. 
inverse Fourier transform vanishes on an interval of the real axis, see Eq. (18). Hence, features commonly exploited to characterize unitary systems, such as the fluctuationdissipation relation itself (see also [59]), might impose qualitative differences between unitary and Lindbladian correlation functions, thus questioning the general usefulness of our result. However, also in these situations, one can take advantage of the constructive nature of the proof of the Theorem by formulating a Lindbladian dynamics such that $C^{U}(t) \approx C^{L}(t)$. The construction of Lemma 1 and Lemma 2 can still be pursued so that one would end up with two unitary dynamics with similar correlation functions, $C^{X}(t) \approx C^{U}(t)$. By applying suitable bounding procedures which apply to unitary dynamics [60], we could then provide a precise bound to the difference between the reduced dynamics $\rho^{U}(t)$ and $\rho^{L}(t)$ based on the difference between their correlation functions.

Conclusions. - In this Letter, we proved in rigorous terms conditions that ensure the equivalence between the two reduced dynamics of an open system as resulting from, respectively, an overall unitary dynamics and the interaction with a simpler environment undergoing a Lindblad evolution. This yields a general nonperturbative way to characterize even highly non-Markovian dynamics with a smaller set of degrees of freedom.

Our result paves the way for rigorous investigations of the validity of the simulation of a unitary dynamics with a computationally simpler Lindblad evolution, possibly dealing with an approximated equality of the two. From a more abstract perspective, our result also represents an extension of the input-output formalism [3] to the bipartite scenario, where the system subjected to the white noise consists of the open system and its non-Markovian core. Indeed, it will be of interest to investigate how this relates to other non-Markovian input-output approaches introduced in the literature [61-63]. In addition, the fact that an input-output formalism can also be developed for a fermionic bath [64] suggests that our result can be extended to this domain. We plan to study such an extension, as well as to treat multitime correlation functions of the open-system observables in order to fully characterize, e.g., the system's spectral response.

We thank Andreas Lemmer for many useful discussions; we acknowledge support by the state of BadenWürttemberg through bwHPC and the German Research Foundation (DFG) through Grant No. INST 40/467-1 FUGG; financial support by the ERC Synergy grant BioQ and the EU project QUCHIP is acknowledged.

[1] H. Carmichael, An Open Systems Approach to Quantum Optics (Springer-Verlag, Berlin, 1993).

[2] H.-P. Breuer and F. Petruccione, The Theory of Open Quantum Systems (Oxford University Press, New York, 2002).
[3] C. W. Gardiner and P. Zoller, Quantum Noise: A Handbook of Markovian and Non-Markovian Quantum Stochastic Methods with Applications to Quantum Optics (Springer, Berlin, 2004).

[4] Á. Rivas and S.F. Huelga, Open Quantum Systems (Springer, New York 2012).

[5] Á. Rivas, S. F. Huelga, and M. B. Plenio, Rep. Prog. Phys. 77, 094001 (2014).

[6] H.-P. Breuer, E. M. Laine, J. Piilo, and B. Vacchini, Rep. Mod. Phys. 88, 021002 (2016).

[7] I. de Vega and D. Alonso, Rep. Mod. Phys. 89, 015001 (2017).

[8] K. K. Metha, C. D. Bruzewicz, R. McConnell, R. J. Ram, J. M. Sage, and J. Chiaverini, Nat. Nanotechnol. 11, 1066 (2016).

[9] J. M. Gambetta, J. M. Chow, and M. Steffen, npj Quantum Inf. 3, 2 (2017).

[10] Y. Wu, F. Jelezko, M. B. Plenio, and T. Weil, Angew. Chem., Int. Ed. Engl. 55, 6586 (2016).

[11] M. Esposito, M. A. Ochoa, and M. Galperin, Phys. Rev. Lett. 114, 080602 (2015).

[12] R. Uzdin, A. Levy, and R. Kosloff, Entropy 18, 124 (2016).

[13] Y. Matsuzaki, S. C. Benjamin, and J. Fitzsimons, Phys. Rev. A 84, 012103 (2011).

[14] A. W. Chin, S. F. Huelga, and M. B. Plenio, Phys. Rev. Lett. 109, 233601 (2012); A. Smirne, J. Kołodyński, S. F. Huelga, and R. Demkowicz-Dobrzański, Phys. Rev. Lett. 116, 120801 (2016).

[15] P. Ribeiro and V. R. Vieira, Phys. Rev. B 92, 100302(R) (2015).

[16] M. T. Mitchison and M. B. Plenio, arXiv:1708.05574.

[17] S. F. Huelga and M. B. Plenio, Contemp. Phys. 54, 181 (2013).

[18] K. M. Pelzer, A. F. Fidler, G. G. Griffin, S. K. Gray, and G. S. Engel, New J. Phys. 15, 095019 (2013).

[19] Y. Tanimura and R. Kubo, J. Phys. Soc. Jpn. 58, 1199 (1989); A. Ishizaki and G. R. Fleming, J. Chem. Phys. 130, 234111 (2009); J. Strümpfer and K. Schulten, J. Chem. Phys. 134, 095102 (2011).

[20] D. E. Makarov and N. Makri, Chem. Phys. Lett. 221, 482 (1994); M. Thorwart, P. Reimann, and P. Hänggi, Phys. Rev. E 62, 5808 (2000); M. Thorwart, E. Paladino, and M. Grifoni, Chem. Phys. 296, 333 (2004).

[21] J. Prior, A. W. Chin, S. F. Huelga, and M. B. Plenio, Phys. Rev. Lett. 105, 050404 (2010); A. W. Chin, Á. Rivas, S. F. Huelga, and M. B. Plenio, J. Math. Phys. 51, 092109 (2010); M. P. Woods, R. Groux, A. W. Chin, S. F. Huelga, and M. B. Plenio, J. Math. Phys. 55, 032101 (2014).

[22] D. Tamascelli, R. Rosenbach, and M. B. Plenio, Phys. Rev. E 91, 063306 (2015).

[23] D. Porras, F. Marquardt, J. von Delft, and J. I. Cirac, Phys. Rev. A 78, 010101(R) (2008).

[24] A. Garg, J. N. Onuchic, and V. Ambegaokar, J. Chem. Phys. 83, 4491 (1985).

[25] R. Martinazzo, B. Vacchini, K. H. Hughes, and I. Burghardt, J. Chem. Phys. 134, 011101 (2011).

[26] P. Strasberg, G. Schaller, N. Lambert, and T. Brandes, New J. Phys. 18, 073007 (2016).

[27] D. Newman, F. Mintert, and A. Nazir, Phys. Rev. E 95, 032139 (2017). 
[28] A. Imamoglu, Phys. Rev. A 50, 3650 (1994).

[29] J. Roden, W. T. Strunz, and A. Eisfeld, J. Chem. Phys. 134, 034902 (2011); J. Roden, W. T. Strunz, K. B. Whaley, and A. Eisfeld, J. Chem. Phys. 137, 204110 (2012).

[30] A. A. Dzhioev and D. S. Kosov, J. Chem. Phys. 135, 174111 (2011).

[31] S. Ajisaka, F. Barra, C. Mejìa-Monasterio, and T. Prosen, Phys. Rev. B 86, 125111 (2012).

[32] S. Mostame, P. Rebentrost, A. Eisfeld, A. J. Kerman, D. I. Tsomokos, and A. Aspuru-Guzik, New J. Phys. 14, 105013 (2012).

[33] F. Schwarz, M. Goldstein, A. Dorda, E. Arrigoni, A. Weichselbaum, and J. von Delft, Phys. Rev. B 94, 155142 (2016); A. Dorda, M. Sorantin, W. von der Linden, and E. Arrigoni, New J. Phys. 19, 063005 (2017).

[34] A. Lemmer, C. Cormick, D. Tamascelli, T. Schaetz, S. F. Huelga, and M. B. Plenio, arXiv:1704.00629.

[35] B. M. Garraway, Phys. Rev. A 55, 2290 (1997); B. M. Garraway ibid. 55, 4636 (1997).

[36] S. Bay, P. Lambropoulos, and K. Mølmer, Phys. Rev. A 57, 3065 (1998); P. Lambropoulos, G. M. Nikolopoulos, T. R. Nielsendag, and S. Bay, Rep. Prog. Phys. 63, 455 (2000).

[37] B. J. Dalton, S. M. Barnett, and B. M. Garraway, Phys. Rev. A 64, 053813 (2001).

[38] S. Maniscalco, F. Francica, R. L. Zaffino, N. Lo Gullo, and F. Plastina, Phys. Rev. Lett. 100, 090503 (2008).

[39] L. Mazzola, S. Maniscalco, J. Piilo, K.-A. Suominen, and B. M. Garraway, Phys. Rev. A 80, 012104 (2009); C. Lazarou, K. Luoma, S. Maniscalco, J. Piilo, and B. M. Garraway, Phys. Rev. A 86, 012331 (2012).

[40] Y. Li, J. Zhou, and Hong Guo, Phys. Rev. A 79, 012309 (2009).

[41] F. F. Fanchini, T. Werlang, C. A. Brasil, L. G. E. Arruda, and A. O. Caldeira, Phys. Rev. A 81, 052107 (2010).

[42] J. Roden, W. T. Strunz, and A. Eisfeld, J. Chem. Phys. 134, 034902 (2011).

[43] Z.-X. Man, A. Smirne, Y.-J. Xia, and B. Vacchini, Phys. Lett. A 376, 2477 (2012); Z.-X. Man, Y.-J. Xia, and R. Lo Franco, Sci. Rep. 5, 13843 (2015).

[44] W.-M. Zhang, P.-Y. Lo, H.-N. Xiong, M. Wei-Yuan Tu, and F. Nori, Phys. Rev. Lett. 109, 170402 (2012).
[45] D. W. Schönleber, A. Croy, and A. Eisfeld, Phys. Rev. A 91, 052108 (2015).

[46] R. P. Feynman and F. L. Vernon, Ann. Phys. (N.Y.) 24, 118 (1963).

[47] N. G. van Kampen, Physica (Utrecht) 74, 215 (1974); N. G. van Kampen, Physica (Utrecht) 74, 239 (1974).

[48] G. Gasbarri and L. Ferialdi, arXiv:1707.06540.

[49] G. Lindblad, Commun. Math. Phys. 48, 119 (1976); V. Gorini, A. Kossakowski, and E. C. G. Sudarshan, J. Math. Phys. 17, 821 (1976).

[50] R. L. Hudson and K. R. Parthasarathy, Commun. Math. Phys. 93, 301 (1984).

[51] K. R. Parthasarathy, An Introduction to Quantum Stochastic Calculus (Birkhäuser, Basel, 1992).

[52] A. Barchielli and M. Gregoratti, Quantum Meas. Quantum Metrol. 1, 34 (2013); A. Barchielli and B. Vacchini, New J. Phys. 17, 083004 (2015).

[53] Lemma 2 essentially means that the quantum regression theorem [1-3,54] holds for the reduced dynamics on $R$, obtained via the partial trace over the unitary on $R-\tilde{E}$.

[54] M. Lax, Phys. Rev. 172, 350 (1968).

[55] See Supplemental Material at http://link.aps.org/ supplemental/10.1103/PhysRevLett.120.030402 for the proofs of Lemma 1, Lemma 2 and of the Theorem.

[56] A. J. Leggett, S. Chakravarty, A. T. Dorsey, M. P. A. Fisher, A. Garg, and W. Zwerger, Rev. Mod. Phys. 59, 1 (1987).

[57] M. Bina, Eur. Phys. J. Spec. Top. 203, 163 (2012).

[58] For the model under consideration with an initial vacuum state of the environment, the correlation spectrum $S(\omega)$ coincides with the environmental spectral density $J(\omega)$ [2].

[59] P. Talkner, Ann. Phys. (N.Y.) 167, 390 (1986).

[60] F. Mascherpa, A. Smirne, S. F. Huelga, and M. B. Plenio, Phys. Rev. Lett. 118, 100401 (2017).

[61] L. Diósi, Phys. Rev. A 85, 034101 (2012).

[62] J. Zhang, Y.-X. Liu, R.-B. Wu, K. Jacobs, and F. Nori, Phys. Rev. A 87, 032117 (2013).

[63] S. Xue, T. Nguyen, M. R. James, A. Shabani, V. Ugrinovskii, and I. R. Petersen, arXiv:1704.00986.

[64] C. W. Gardiner, Opt. Commun. 243, 57 (2004). 